\title{
EXPERT
REVIEWS
}

\section{Tackling the human adipose tissue proteome to gain insight into obesity and related pathologies}

Expert Rev. Proteomics 6(4), xxx-xxx (2009)

\section{Belén Peral', Emilio Camafeita, José-Manuel Fernández-Real and Juan Antonio López ${ }^{+}$Author for correspondence Instituto de Investigaciones Biomédicas, Alberto Sols, CSIC \& Universidad Autónoma de Madrid, Arturo Duperier, 4, E-28029 Madrid, Spain Tel.: +34915854 478 Fax: +34915854 401 bperal@iib.uam.es}

Obesity is becoming an important public health problem given its strong association with insulin resistance and Type 2 diabetes. Previously considered an inert depot, fat is now regarded as a highly metabolically active tissue in many pathophysiological processes. In humans, the accumulation of omental rather than subcutaneous adipose tissue appears to be tightly linked to cardiovascular disease and other important comorbidities. Proteomics has emerged as a method for the large-scale study of proteins in biological samples, for instance, fluids, cells or tissues, which encompasses not only the identities of the proteins present, but also quantification and post-translational modification events. Human adipose tissue proteome analysis, still in its early stages, may help understand the molecular mechanisms of obesity and the role of omental fat in the pathogenesis of obesity-associated diseases. This review covers recent advances in human adipose tissue proteomics, focusing on the analysis of the omental and the subcutaneous fat.

KeYwords: 2D fluorescence difference gel electrophoresis $\bullet$ adipose tissue $\bullet$ metabolic syndrome $\bullet$ mass spectrometry $\bullet$ obesity $\bullet$ omental fat $\bullet$ polycystic ovary syndrome $\bullet$ proteomics $\bullet$ secretome $\bullet$ subcutaneous fat

Obesity is recognized as one of the most important public health problems facing the world today. According to the WHO, more than one billion adults are overweight, and 300 million are clinically obese. Prevalence is growing exponentially worldwide, both in developed and developing countries [1]. Of particular concern, obesity has also increased dramatically over the last decades in children and adolescents [2].

Obese subjects have a decreased quality of life and life expectancy, as well as an increased risk of suffering insulin resistance, Type 2 diabetes, cardiovascular disease (CVD), hepatic steatosis, pulmonary and muscular pathologies, psychological disorders, and cancer, among others. The authors of a recent systematic review searched reports to identify prospective studies of incident cases of 20 cancer types, including 282,137 incident cases. Associations were generally similar in studies from North America, Europe and Australia, and the Asia-Pacific region. Increased BMI was associated with increased risk of common and less common malignancies [3].

The physiologic processes that regulate weight and metabolism, including peripheral hunger and satiety signals, the central integration of this information, and the integrated gastrointestinal response to food intake, have received intense investigation, particularly during the past decade. A person's weight and body composition are likely determined by interaction between his/ her genetic makeup and social, cultural, behavioral and environmental factors. An increased intake of energy-dense foods, especially when combined with reduced physical activity, surely contributes to the high prevalence of obesity; however, the existence of complex systems that regulate energy balance requires that this paradigm be considered in a larger context [4].

Prevention, treatment and understanding of obesity etiology should be a priority for both scientific and government communities in view that this disease and its associated comorbidities are a major economic and societal burden.

\section{Human adipose tissue: omental \& subcutaneous fat biology}

In humans, the adipose tissue is dispersed throughout the body with major intra-abdominal depots around the omentum, intestines and 


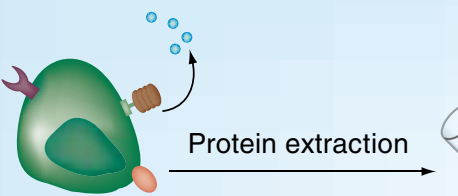

Tissue, cell, fluid...

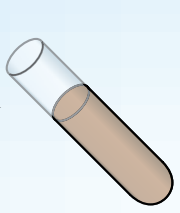

Protein mixture
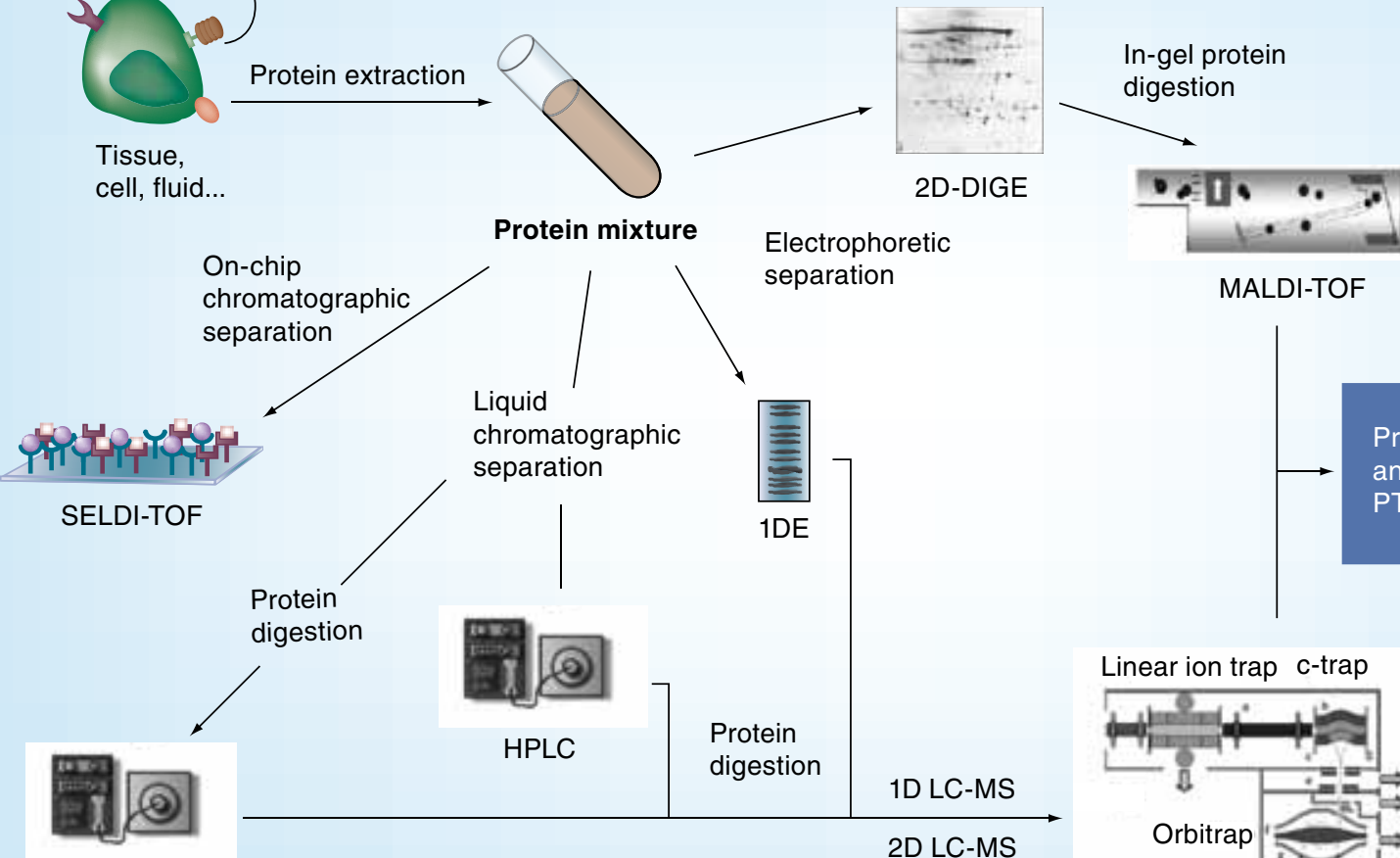

nHPLC

On-chip hromatographic eparation
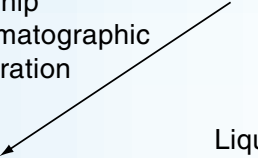

Liquid chromatographic separation

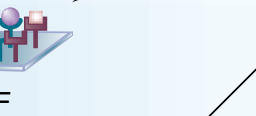$$
\text { (1) }
$$

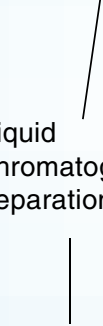

rotein

digestion
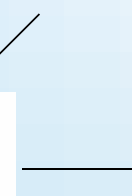

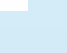

separation
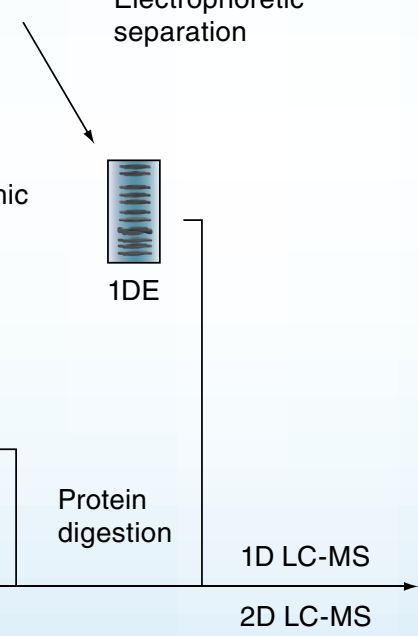

2D LC-MS
MALDI-TOF
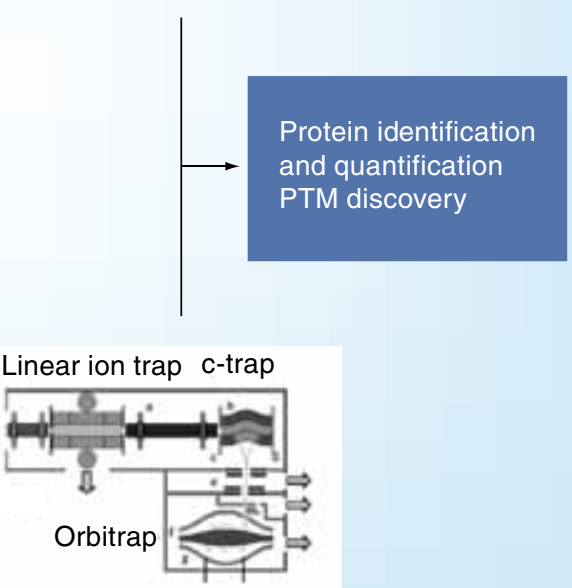

ESI-LIT Orbitrap

Figure 1. Representative proteomic strategies. Two major proteomic workflows are shown, based on electrophoretic and chromatographic separation. Proteins extracted from biological samples can be separated according to either their Mw (1DE) or both their Mw and pl (2D-DIGE). Proteins, and especially peptides thereof produced by proteolysis, can be complimentarily separated by LC. After in-solution or in-gel enzymatic digestion of the proteins, the tryptic products are analyzed by MS. Protein identification is achieved by interrogating protein databases with MS data from peptide mass fingerprints and/or MS/MS data from peptide fragmentation fingerprints. As an alternative, protein extracts can be separated on solid-state chromatographic surfaces and the retained proteins can be mass analyzed in situ for protein profiling.

1DE: 1D gel electrophoresis; 2D-DIGE: 2D difference gel electrophoresis; ESI: Electrospray ionization; HPLC; High-performance liquid chromatography; LC: Liquid chromatography; LIT: Linear ion trap; MS: Mass spectrometry; MS/MS; Tandem mass spectrometry; Mw: Molecular weight; pl: Isoelectric point.

perirenal areas, as well as in subcutaneous depots in the buttocks, thighs and abdomen. Some of these depots, known as omental or visceral fat, drain directly into the portal circulation and have been linked to many of the morbidities associated with obesity, including Type 2 diabetes and CVD [5]. In addition, adipose tissue can be found in many other areas fulfilling a function of mechanical support, including the retro-orbital space, fat pads of the heels, fingers and toes, and the bone marrow. Fat distribution, even in thin individuals with steady bodyweight and stable BMI, changes with age, decreasing in retro-orbital fat and subcutaneous fat and increasing in intra-abdominal fat [6,7].

Adipose tissue, previously seen as an inert fat depot, is now recognized as a key, highly active and versatile organ with many important physiological and pathological roles. Besides adipocytes, adipose tissue contains connective tissue matrix, nerve tissue, stromal-vascular (SV) cells, and immune cells. Most of the adipose tissue functions are carried out via molecules secreted by adipocytes, macrophages and other fat cells, capable of acting locally and in many nonadipose tissues, such as muscle, liver and stomach tissues, as well as on nervous, immune and vascular systems, and even on bone turnover [4]. Therefore, fat is an endocrine organ which cross-talks with other essential organs like the liver, the muscle, the pancreas and the brain, being a crucial regulator of whole-body homeostasis.

\section{Proteomics technologies}

Proteomics is the large-scale study of proteins, including their interactions, post-translational modifications (PTM), localization and functions [8]. A collection of proteomic approaches employing multiple methodological strategies and technological platforms are available for protein detection, identification and characterization in disease-related processes, at the whole cell or tissue level, and even in subcellular structures and in biological fluids (FIGURE 1). 


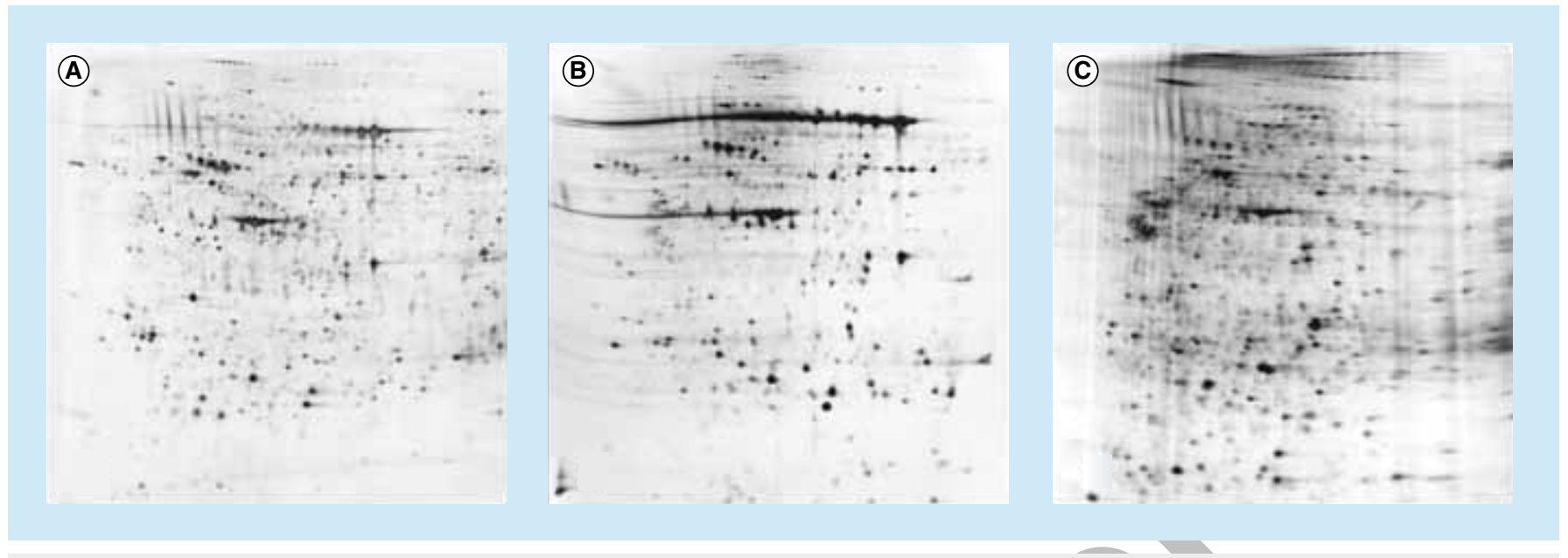

Figure 2. 2D protein separation of human omental adipose tissue. Adipose tissue proteins were solubilized with the Partial Mammalian Proteome Extraction Kit (Calbiochem), and a total of $100 \mu \mathrm{g}$ of protein were loaded onto 4-7 pH strips in the first dimension. For the second dimension, SDS-PAGE with $12 \%$ polyacrylamide was used, and proteins were visualized by silver staining. (A) Whole tissue extract. (B) Highly soluble proteins (fraction 1). (C) Proteins with intermediate solubility (fraction 2).

\section{Protein separation}

Most protein separation approaches are based on electrophoretic or chromatographic methods. The classical approach for the quantitative profiling of protein expression has relied on $2 \mathrm{DE}$ in combination with mass spectrometry (MS) [9]. An important advance in 2DE is the fluorescence difference gel electrophoresis (DIGE) system, in which two pools of samples are labeled with different fluorescent dyes, mixed and subjected to separation in the same 2DE gel [10]. This technology allows reliable quantification of differences in protein expression between two conditions with minimal technical variability.

Complementary gel-free schemas for proteomic analysis rely on liquid chromatography (LC) separation of proteins or peptides [11], with or without isotopic or isobaric labeling [12]. An important implementation of LC, the multidimensional protein identification technology, is based on the combination of two stationary phases for increased resolution in the separation of complex peptide mixtures [13]. Usually, these chromatographic technologies are online-coupled to mass spectrometry (LC-MS) for automated protein identification, characterization of PTMs, and protein quantification.

\section{Protein identification \& quantification}

Owing to its superior sensitivity and selectivity, MS is the analytical technique of choice for protein identification. MS instruments ionize and vaporize the analytes using different ionization techniques, notably ESI and MALDI. These ions are then separated and detected in the gas phase within the mass analyzer, which is normally based on electric fields (e.g., TOF, ion traps or Orbitrap) or magnetic fields (e.g., FT-ICR). For protein identification and characterization, proteins are usually digested with trypsin. The masses of the resulting peptides, or fragments thereof, are accurately measured by MS, and these experimental values are matched against theoretical values calculated from sequence databases. Isotopic or isobaric labeling of peptides is the widest used methodology for quantification in gel-free approaches [12].

\section{Proteomics of human adipose tissue}

Despite that proteomic-based approaches are widely used to study a variety of human tissues relating to different disorders, the proteomic analysis of tissues presents some challenging hurdles. Tissues are heterogeneous as they are comprised of multiple cell types and are difficult to obtain in sufficient quantities. Moreover, to minimize technical variability for comparative studies, it is essential to train clinical staff to collect samples from similar areas, across all patients treated with identical surgery procedures, and rapidly freeze biopsies at $-80^{\circ} \mathrm{C}$ to avoid protein degradation.

Adipose tissue proteomic studies present additional difficulties due to its large content in triglycerides (often more than $50 \%$ of the tissue volume), which interferes with protein solubilization and separation, and albumin, both of which hamper subsequent 2DE analysis. To deplete albumin and other abundant proteins, we have assayed some ligand-affinity methods such as the albumin and IgG removal kit (GE Healthcare), the montage albumin deplete kit (Millipore), and the mimetic screening column kit (Prometic Biosciences). However, as albumin is a carrier/transport protein that binds other important molecules, many other proteins were removed upon treatment (Peral etal., unpublished). This fact, together with the reduced 2DE reproducibility, discouraged us to follow this approach.

We have applied fractionation tools to reduce the complexity of adipose tissue protein mixtures based on reagents with differential solubilization properties (partial mammalian proteome extraction kit, Calbiochem). We analyzed two fractions: one containing very soluble proteins (fraction 1) and another containing proteins with intermediate solubility (fraction 2). Serum albumin was kept in fraction 1, as shown in Figure 2. This approach increases the number of spots detected, at the expense of doubling the amount of biopsy required, as well as the number of gels to be analyzed.

Due to the aforementioned limitations, only a few reports have addressed the proteomic investigation of human adipose tissue. A pioneer study carried out in 2004 by our group [14], described the 


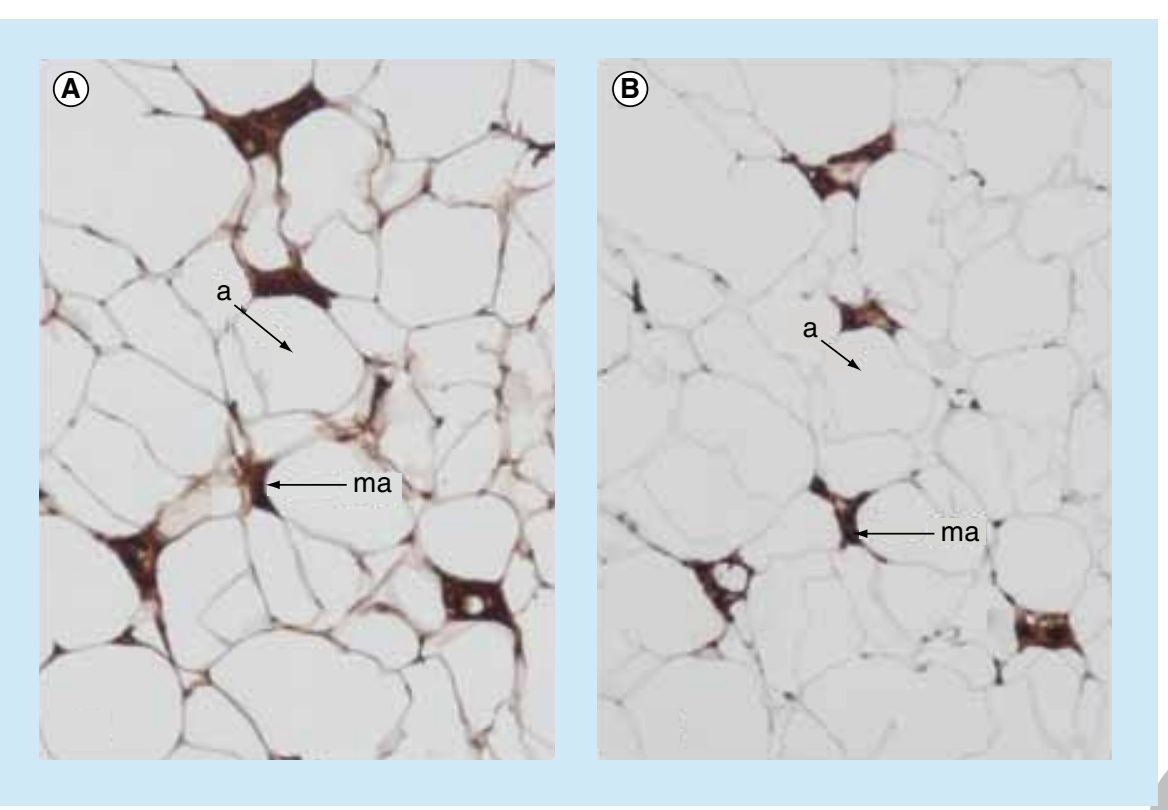

Figure 3. Expression of FABP5 protein in the omental adipose tissue.

Immunohistochemical detection (brown) of (A) FABP5 and (B) macrophage-specific antigen CD68, in omental fat from a morbidly obese patient. Images are representative of adipose tissue sections collected from five subjects. a, adipocyte; ma, macrophage.

Some of the dysregulated proteins identified were confirmed by immunoblotting and immunolocalization assays. In spite of the high fat content of adipose tissue, immunohistochemical and immunofluorescence analyses could be successfully performed to find out the distribution of specific proteins in the adipose tissue. One of the proteins studied has been the fatty acid-binding protein, epidermal FABP5, which plays an important role in the adipose tissue for transporting not only fatty acids resulting from adipocyte lipolysis, but also retinoic acid into the nucleus favoring survival pathways [25]. Immunohistochemical analysis revealed FABP5 expression in adipocytes as well as in nonfat cells issuing from the SV fraction. Some of these SV cells were found to be macrophages, as shown by the CD68 labeling (Figure 3). Recently a few reports have shown an increased infiltration of macrophages in the omental fat, compared with the subcutaneous fat $[26,27]$, which could account for the overexpression of FABP5 in

optimization of a protocol for protein extraction, and improved focusing in the alkaline region of $2 \mathrm{DE}$ maps based on cup-loading and DTT replacement by hydroxyethyl disulfide [15] in the strip rehydration solution. This led to the first description of the human adipose tissue proteome [14].

\section{Differential expression of proteins in omental \& subcutaneous adipose tissue}

The size of the omental, more than the subcutaneous, fat is strongly related to a higher risk of suffering insulin resistance, Type 2 diabetes, dyslipidemia and CVD, among other diseases and comorbidities [5,16]; this is probably due to their dissimilar gene expression patterns and different biochemical, cellular and metabolic properties [17-23]. Recently, our group has investigated the difference between the omental and subcutaneous human adipose tissue proteome from healthy obese patients $\left(30>\mathrm{BMI}<40 \mathrm{~kg} / \mathrm{m}^{2}\right)$ by $2 \mathrm{D}$-DIGE and MS, together with immunoblotting and immunolocalization analysis [24]. This study revealed 43 differentially expressed proteins and underlined the high metabolic activity of the omental compared with the subcutaneous adipose tissue, since a set of proteins which were upregulated in the omental fat were engaged in such metabolic pathways as carbohydrate and lipid metabolism, oxidation-reduction, lipid transport, protein synthesis, protein folding, cellular stress and inflammation. Despite that some of these deregulated proteins had been previously identified in studies based on animal models or in vitro adipocytes [24], most of these proteins represented newly described molecules involved in the pathophysiology of obesity and/ or associated disorders, thus opening up new possibilities in the study of these diseases. Given the novelty of some of these proteins, they have not undergone appropriate biological follow-up yet and the study of their functional role is underway in our laboratory. the omental fat from obese subjects. The infiltrated adipose tissue macrophages localize to 'crown-like structures' around individual adipocytes and are widespread in the omental fat of morbidly obese individuals [28]. Figure 4 shows the co-localization of FABP5 and CD68 in an omental fat cryosection from a morbidly obese patient.

In addition, we have used these strategies to confirm the presence of epithelial cytokeratins (CK-8, CK-7, CK-18 and CK-19) in the omental adipose tissue [24], the only fat tissue presenting mesothelium sheets surrounding the fat lobules (FIGure 5) [29,30]. In our laboratory these cytokeratins are routinely employed to differentiate omental from subcutaneous fat samples. We have hypothesized that omental mesothelial cells might increase the mechanical stress of hypertrophied adipocytes during obesity development, producing cellular stress, which, in turn, would activate the unfolded protein response (UPR) signaling pathway. Of note, mouse model studies have suggested that UPR not only interferes with the insulin signaling pathway, but is also involved in initiating inflammation [31].

\section{Proteomics of omental adipose tissue in other obesity-related diseases}

As mentioned previously, the omental fat has been associated with a higher risk for suffering abdominal obesity-related comorbidities. Polycystic ovary syndrome (PCOS) is a complex endocrine disorder with a high prevalence (6-7\%) in premenopausal women [32]. Androgen excess is the central defect in PCOS, leading to hyperandrogenism, oligoovulation and/or polycystic ovarian morphology [33]. Obesity plays a relevant role in the development of PCOS. More that $40 \%$ of PCOS patients are overweight or obese in an American population [34]. On the other hand, PCOS is probably the most frequent association of obesity in premenopausal women [35]. This relation may be explained by adipose tissue 
secretion of several molecules that favor the insulin resistant and low-grade chronic inflammatory state characteristic of PCOS $[36,37]$. It has been reported that weight loss in morbidly obese PCOS patients, after bariatric surgery, resolves PCOS signs and symptoms, and improves insulin sensitivity and other metabolic comorbidities, underlining the importance of abdominal adiposity in PCOS [38]. Considering the relevant function that adipose tissue plays in PCOS pathogenesis, our group has focused not only on proteomic, but also on transcriptomic analysis to study omental fat from morbidly obese women presenting with or without PCOS $[39,40]$. The transcriptomic studies, based on the microarray technology (GeneChips from Affymetrix), revealed changes in the expression profiles of 63 genes, related mainly with insulin- and Wntsignaling, lipid metabolism, oxidative stress, inflammation and immune response, along with other genes previously related to obesity and related comorbidities [39].

The comparative proteomic study utilized 2D-DIGE and MALDI-MS [40], to uncover differences in the expression of proteins involved mainly in lipid and glucose metabolism, oxidative stress and adipocyte differentiation. Interestingly, both the transcriptomic and proteomic studies revealed the overexpression of glutathione $S$-transferase $\mathrm{Mu}-3$ in the omental fat of PCOS women, illustrating the complementariness of these approaches. It is noteworthy that both works have not only confirmed the well-known alteration of the insulin-signaling pathway, but also revealed novel functional pathways related to inflammation and oxidative stress, among others, in agreement with previous studies on PCOS [41-43].

Another fat depot, the mammary adipose tissue, has been analyzed by $2 \mathrm{DE}, \mathrm{MS}$, antibody arrays and immunoblotting [44]. The authors identified 359 proteins from breast cancer patients involved in many biological processes including signal transduction, cell communication, energy metabolism, immune response, transport and apoptosis. This report offered for the first time an overview of the mammary fat proteome and its interstitial fluid, emphasizing the significant role of the adipocyte in the breast tumor microenvironment.

\section{Proteomics of human adipogenesis}

Two works have resorted to $2 \mathrm{DE}$ and MS to study human adipogenesis in vitro. Human adipose-derived adult stem (ADAS) cells were isolated by collagenase digestion of subcutaneous fat aspirates from lean subjects [45]. Protein extracts were compared before and after adipocyte differentiation from ADAS cells and a set of proteins were found modulated, showing the relevance of the heat-shock protein (HSP) family in the adipogenesis process. Noteworthy, our comparative proteomic study in omental and subcutaneous fat also revealed the differential expression of three HSPs (HSP90, HSP70 and HSP27) [24]. HSPs have been involved in obesity, insulin resistance and diabetes [31,46,47], while HSP70 has been genetically associated with obesity and Type 2 diabetes in case-control studies [48,49]. Lee et al. focused on proteins that were differentially expressed by the differentiation of human mesenchymal stem cells into adipocytes, revealing four proteins associated with adipogenesis [50].

\section{Proteomics of human adipose tissue secretome}

Since most of the functions exerted by the adipose tissue are mediated by secreted proteins, the study of the secretome is of particular interest, and has been approached by a variety of proteomic methods. In primary human adipocytes isolated from omental fat tissue, the combined use of 2DE and MS allowed the identification of several serine protease inhibitors (serpins) [51]. Omental adipose tissue explants were cultured in media 


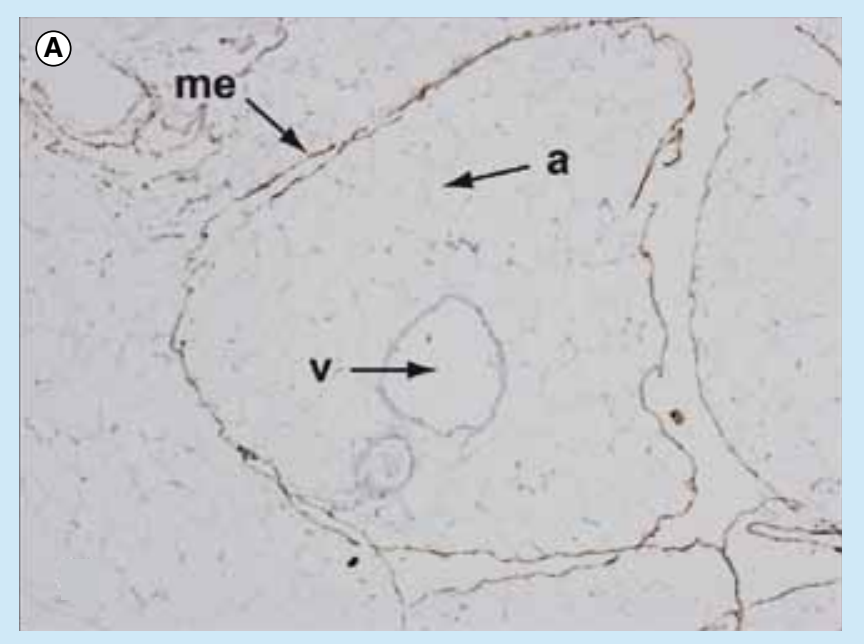

Figure 5. Immunohistochemical detection of CK-18 in omental adipose tissue with anti-CK-18 antibody. Only the mesothelial cells enveloping fat lobules are specifically stained with anti-CK-18 antibody in brown (A). The immunostaining with the secondary antibody alone is also shown (B). Images are representative of adipose tissue preparations collected from five subjects.

a: Adipocyte; me: Mesothelium; v: Vessel.

containing L- $\left[{ }^{13} \mathrm{C}_{6},{ }^{15} \mathrm{~N}_{2}\right]$ lysine, followed by SDS-PAGE fractionation and protein identification based on LC-MS [52]. A total of 70 of the proteins identified incorporated the label and were considered true adipose tissue-derived components related with signaling, extracellular matrix, immune function and degradation functional categories. Moreover, Klimcakova et al. made use of a human cytokine antibody array to provide an overview of the proteins secreted by human subcutaneous adipose tissue, in particular to investigate the effect of PPAR agonists on the production of adipokines [53]. Results showed that PPAR ligands regulate adipokine secretion. The same antibody array was utilized to search for novel adipokines abnormally secreted by omental adipose tissue in obesity [54]. The analysis of factors from isolated adipocytes and SV fraction, from obese and nonobese men, identified six new adipokines increased in obesity. These molecules were associated with several features of the metabolic syndrome, supporting the link between obesity and its cardiovascular or metabolic comorbidities.

\section{Expert commentary}

Human obesity is a very complex dysfunction, which results from the interaction between the individual's genetic background and his or her environmental, social and cultural factors. Fat, formerly considered an inert depot, is now recognized as a highly active organ, orchestrating vital physiological roles by secreting a wide variety of molecules that influence many body tissues. Numerous proteomics approaches have been applied with different animal models and cultured cells (see an excellent review by Barceló-Batllori et al. [55]), but only a few works have examined the protein content of the human adipose tissue, or the identification of the adipose tissue secretome. These techniques have been also employed to unveil protein expression differences between omental and subcutaneous fat depots, as the omental tissue is tightly linked to obesity-related disorders. The information attained from proteomic studies on human adipose tissue, the topic of this special report, is providing crucial information to understand indepth the pathophysiology associated with enlarged adipose tissue.

\section{Five-year view}

These are exciting times for all those working in obesity and proteomics. The next 5 years will be a crucial time for applying proteomics capacities to gain insight into obesity and related pathologies, as well as for translating this knowledge to limit spreading of these disturbing disorders.

The mechanistic links between obesity and its comorbidities will be a fertile area to study by proteomics approaches. Fat is now recognized as a highly active cross-talking tissue with important physiological and pathological roles. Numerous adipose tissue proteins have been discovered associated with obesity, but probably other molecules are still awaiting discovery. Characterizing these products, studying their function and interaction with other tissues, and further exploring their effects will increase our knowledge about how fat excess is contributing to the pathogenesis of both established metabolic syndrome abnormalities and unknown obesity-related diseases. The comprehensive study by proteomics technologies of the fat cells and their secreted products, including the precursor cells, will help us to understand phenotypic changes of the adipose tissue induced by obesity. Moreover, these studies will shed light on how adipose tissue responds to changes in energy balance, and most important, how to devise anti-obesity therapies. Most of this work will require the identification of low-abundance proteins and detection of very subtle expression changes under obesity conditions, a difficult task with currently available proteomics tools. A combination of multiple approaches, including new instrumentation, such as the Orbitrap, to increase sensitivity and proteome coverage will be needed. Improved fractionation methods and the analysis of specific subproteomes, for example, the phosphoproteome, will 
render a deeper view of the fat cells proteome. However, the central step will be the leap from a static proteome analysis to quantitative proteomics experiments, focusing on the dynamics of each protein and their closest interacting partners, which will provide unprecedented dynamic views of the entire cell proteome.

\section{Financial \& competing interests disclosure}

This work was supported by Grants SAF-2006-02354 and SAF-200802073 from the Ministerio de Educacion y Ciencia, Spain. We thank
Rafael Pérez-Pérez, Eva Garcia-Santos and Francisco Ortega-Delgado for their technical help. The CNIC is supported by the Ministerio de Ciencia e Innovación and the Fundacion Pro CNIC. CIBERobn is an initiative from the Instituto de Salud Carlos III. The authors have no other relevant affiliations or financial involvement with any organization or entity with a financial interest in or financial conflict with the subject matter or materials discussed in the manuscript apart from those disclosed.

No writing assistance was utilized in the production of this manuscript.

\section{Key issues}

- Obesity predisposes to Type 2 diabetes, cardiovascular disease, hepatic steatosis, hypertension, pulmonary and muscular pathologies, cancer, and psychological disorders among other diseases.

- The adipose tissue is an important endocrine organ which cross-talks with other central organs like the liver, the muscle, the pancreas, and the brain, acting as a crucial regulator of whole-body homeostasis.

- Omental adipose tissue is more tightly linked to obesity-associated comorbidities, like cardiovascular disease and metabolic syndrome, than the subcutaneous fat. This is likely due to differences in the production of adipokines and fatty acids released from omental fat into the portal circulation.

- The adipose tissue, due to its high fat and albumin content, exhibits particular difficulties for proteome analysis.

- Proteomics characterization of signaling pathways in adipose tissue cells is pivotal for understanding the biological mechanism underlying obesity and its comorbidities.

- Prevention of childhood obesity by shifting obesogenic lifestyle habits from the infant age must be an important public health priority.

\section{References}

Papers of special note have been highlighted as: - of interest

-. of considerable interest

1 Haslam DW, James WP. Obesity. Lancet 366(9492), 1197-1209 (2005).

2 Daniels SR, Jacobson MS, McCrindle BW, Eckel RH, Sanner BM. American Heart Association childhood obesity research summit report. Circulation 119(15), e489e517 (2009).

3 Renehan AG, Tyson M, Egger M, Heller $\mathrm{RF}$, Zwahlen M. Body-mass index and incidence of cancer: a systematic review and meta-analysis of prospective observational studies. Lancet 371(9612), 569-578 (2008).

-. Association between obesity and cancer (both common and uncommon types).

4 Spiegelman BM, Flier JS. Obesity and the regulation of energy balance. Cell 104(4), 531-543 (2001).

5 Matsuzawa Y. Therapy insight: adipocytokines in metabolic syndrome and related cardiovascular disease. Nat. Clin. Pract. Cardiovasc. Med.3(1), 35-42 (2006).

6 Rosen ED, Spiegelman BM. Adipocytes as regulators of energy balance and glucose homeostasis. Nature 444(7121), 847-853 (2006).

7 Gesta S, Tseng YH, Kahn CR. Developmental origin of fat: tracking obesity to its source. Cell 131(2), 242-256 (2007).
-• The origin of adipocytes within adipose tissue.

8 Dominguez DC, Lopes R, Torres ML. Proteomics: clinical applications. Clin. Lab. Sci. 20(4), 245-248 (2007).

9 Hanash S. 2-D or not 2-D - is there a future for 2-D gels in proteomics? Insights from the York proteomics meeting. Proteomics 1(5), 635-637 (2001).

10 Unlu M, Morgan ME, Minden JS. Difference gel electrophoresis: a single gel method for detecting changes in protein extracts. Electrophoresis 18(11), 2071-2077 (1997).

- First paper on the optimization of a new methodology for 2D electrophoresis by using fluorocromes.

11 Boysen RI, Hearn MT. HPLC of peptides and proteins. In: Current Protocols in Protein Science (Chapter 8). John Wiley \& Sons, Inc., NJ, USA (2001).

12 Ross PL, Huang YN, Marchese JN et al. Multiplexed protein quantitation in Saccharomyces cerevisiae using aminereactive isobaric tagging reagents. Mol. Cell. Proteomics 3(12), 1154-1169 (2004).

- Development of the iTRAQ labeling technology for quantitative proteomics analysis.

13 Liao H, Wu J, Kuhn E et al. Use of mass spectrometry to identify protein biomarkers of disease severity in the synovial fluid and serum of patients with rheumatoid arthritis. Arthritis Rheum. 50(12), 3792-3803 (2004).

14 Corton M, Villuendas G, Botella JI, San Millan JL, Escobar-Morreale HF, Peral B. Improved resolution of the human adipose tissue proteome at alkaline and wide range $\mathrm{pH}$ by the addition of hydroxyethyl disulfide. Proteomics 4(2), 438-441 (2004).

- Pioneering work in the optimization of a protocol to extract, separate and identify human adipose tissue proteins.

15 Olsson I, Larsson K, Palmgren R, Bjellqvist $B$. Organic disulfides as a means to generate streak-free two-dimensional maps with narrow range basic immobilized $\mathrm{pH}$ gradient strips as first dimension. Proteomics 2(11), 1630-1632 (2002).

16 Fox CS, Massaro JM, Hoffmann U et al. Abdominal visceral and subcutaneous adipose tissue compartments: association with metabolic risk factors in the framingham heart study. Circulation 116(1), 39-48 (2007).

- Association of the omental fat with a major risk of suffering obesity-related diseases.

17 Ramis JM, Franssen-van Hal NL, Kramer $\mathrm{E}$ et al. Carboxypeptidase E and thrombospondin-1 are differently expressed in subcutaneous and visceral fat of obese subjects. Cell. Mol. Life Sci. 59(11), 1960-1971 (2002). 
18 Gabrielsson BG, Johansson JM, Lonn M et al. High expression of complement components in omental adipose tissue in obese men. Obes. Res. 11(6), 699-708 (2003).

19 Linder K, Arner P, Flores-Morales A, Tollet-Egnell P, Norstedt G. Differentially expressed genes in visceral or subcutaneous adipose tissue of obese men and women. $J$. Lipid Res. 45(1), 148-154 (2004).

20 Vohl MC, Sladek R, Robitaille J et al. A survey of genes differentially expressed in subcutaneous and visceral adipose tissue in men. Obes. Res. 12(8), 1217-1222 (2004).

21 Gesta S, Bluher M, Yamamoto Y et al. Evidence for a role of developmental genes in the origin of obesity and body fat distribution. Proc. Natl. Acad. Sci. USA 103(17), 6676-6681 (2006).

- Overview of fat depot differences in gene expression highlighting genes involved in embryonic development pattern specification.

22 van Beek EA, Bakker AH, Kruyt PM, Hofker MH, Saris WH, Keijer J. Intra- and interindividual variation in gene expression in human adipose tissue. Pflugers Arch. 453(6), 851-861 (2007).

23 MacLaren R, Cui W, Simard S, Cianflone $\mathrm{K}$. Influence of obesity and insulin sensitivity on insulin signaling genes in human omental and subcutaneous adipose tissue. J. Lipid Res. 49(2), 308-323 (2008).

24 Perez-Perez R, Ortega-Delgado FJ, Garcia-Santos E et al. Differential proteomics of omental and subcutaneous adipose tissue reflects their unalike biochemical and metabolic properties. J. Proteome Res. 8(4), 1682-1693 (2009).

- First publication about the identification of differentially expressed proteins in human omental versus subcutaneous fat using proteomic approaches.

25 Schug TT, Berry DC, Shaw NS, Travis SN, Noy N. Opposing effects of retinoic acid on cell growth result from alternate activation of two different nuclear receptors. Cell 129(4), 723-733 (2007).

26 Cancello R, Tordjman J, Poitou C et al. Increased infiltration of macrophages in omental adipose tissue is associated with marked hepatic lesions in morbid human obesity. Diabetes 55(6), 1554-1561 (2006).

-• Describes the increased infiltration of macrophages in the morbidly obese omental fat.

27 Harman-Boehm I, Bluher M, Redel H et al. Macrophage infiltration into omental versus subcutaneous fat across different populations: effect of regional adiposity and the comorbidities of obesity. J. Clin. Endocrinol. Metab. 92(6), 2240-2247 (2007).

28 Cinti S, Mitchell G, Barbatelli G et al. Adipocyte death defines macrophage localization and function in adipose tissue of obese mice and humans. J. Lipid Res. 46(11), 2347-2355 (2005).

- Describes the localization of macrophages in obese adipose tissue.

29 Wilkosz S, Ireland G, Khwaja N et al. A comparative study of the structure of human and murine greater omentum. Anat. Embryol. 209(3), 251-261 (2005).

30 van Hinsbergh VW, Kooistra T, Scheffer MA, Hajo van Bockel J, van Muijen GN. Characterization and fibrinolytic properties of human omental tissue mesothelial cells. Comparison with endothelial cells. Blood 75(7), 1490-1497 (1990).

31 Ozcan U, Cao Q, Yilmaz E et al. Endoplasmic reticulum stress links obesity, insulin action, and Type 2 diabetes. Science 306(5695), 457-461 (2004).

- Focuses on the correlation of endoplasmic reticulum stress and the activation of unfolded protein response signaling pathway when suffering obesity-related disorders.

32 Asuncion M, Calvo RM, San Millan JL, Sancho J, Avila S, Escobar-Morreale HF. A prospective study of the prevalence of the polycystic ovary syndrome in unselected caucasian women from Spain. J. Clin. Endocrinol. Metab. 85(7), 2434-2438 (2000).

33 Azziz R, Carmina E, Dewailly D et al. Positions statement: criteria for defining polycystic ovary syndrome as a predominantly hyperandrogenic syndrome: an Androgen Excess Society guideline. J. Clin. Endocrinol. Metab. 91(11), 4237-4245 (2006).

34 Azziz R, Woods KS, Reyna R, Key TJ, Knochenhauer ES, Yildiz BO. The prevalence and features of the polycystic ovary syndrome in an unselected population. J. Clin. Endocrinol. Metab. 89(6), 2745-2749 (2004).

35 Alvarez-Blasco F, Botella-Carretero JI, San Millan JL, Escobar-Morreale HF. Prevalence and characteristics of the polycystic ovary syndrome in overweight and obese women. Arch. Intern. Med. 166(19), 2081-2086 (2006).

36 Fernandez-Real JM, Ricart W. Insulin resistance and chronic cardiovascular inflammatory syndrome. Endocr. Rev. 24(3), 278-301 (2003).

- Focuses on low-level and chronic inflammatory condition of obesity and obesity-related diseases.

37 Escobar-Morreale HF, Luque-Ramirez M, San Millan JL. The molecular-genetic basis of functional hyperandrogenism and the polycystic ovary syndrome. Endocr. Rev. 26, 251-282 (2005).

- Focuses on the polycystic ovary syndrome as a complex disease, summarizing numerous genomic variants associated to the multiple phenotypes of the disorder.

38 Escobar-Morreale HF, Botella-Carretero JI, Alvarez-Blasco F, Sancho J, San Millan JL. The polycystic ovary syndrome associated with morbid obesity may resolve after weight loss induced by bariatric surgery. $J$. Clin. Endocrinol. Metab. 90 (12), 63646369 (2005).

39 Corton M, Botella-Carretero JI, Benguria A et al. Differential gene expression profile in omental adipose tissue in women with polycystic ovary syndrome. J. Clin. Endocrinol. Metab. 92(1), 328-337 (2007).

- The differences in gene expression in obese women with and without polycystic ovary syndrome, highlighting several signaling pathways in addition to insulin signaling as associated to the disease.

40 Corton M, Botella-Carretero JI, Lopez JA et al. Proteomic analysis of human omental adipose tissue in the polycystic ovary syndrome using two-dimensional difference gel electrophoresis and mass spectrometry. Hum. Reprod. 23(3), 651-661 (2008).

- Focuses on the omental adipose tissue proteome in polycystic ovary syndrome.

41 Fenkci V, Fenkci S, Yilmazer M, Serteser M. Decreased total antioxidant status and increased oxidative stress in women with polycystic ovary syndrome may contribute to the risk of cardiovascular disease. Fertil. Steril. 80(1), 123-127 (2003).

42 Sabuncu T, Vural H, Harma M, Harma M. Oxidative stress in polycystic ovary syndrome and its contribution to the risk of cardiovascular disease. Clin. Biochem. 34(5), 407-413 (2001).

43 Kelly CC, Lyall H, Petrie JR, Gould GW, Connell JM, Sattar N. Low grade chronic inflammation in women with polycystic ovarian syndrome. J. Clin. Endocrinol. Metab. 86(6), 2453-2455 (2001).

44 Celis JE, Moreira JM, Cabezon T et al. Identification of extracellular and 
intracellular signaling components of the mammary adipose tissue and its interstitial fluid in high risk breast cancer patients: toward dissecting the molecular circuitry of epithelial-adipocyte stromal cell interactions. Mol. Cell. Proteomics 4(4), 492-522 (2005).

-. Shows the identification of numerous mammary adipose tissue proteins from breast cancer patients.

45 DeLany JP, Floyd ZE, Zvonic S et al. Proteomic analysis of primary cultures of human adipose-derived stem cells: modulation by adipogenesis. Mol. Cell. Proteomics 4(6), 731-740 (2005).

46 Chung J, Nguyen AK, Henstridge DC et al. HSP72 protects against obesity-induced insulin resistance. Proc. Natl. Acad. Sci. USA 105(5), 1739-1744 (2008).

47 Kurucz I, Morva A, Vaag A et al. Decreased expression of heat shock protein 72 in skeletal muscle of patients with Type 2 diabetes correlates with insulin resistance. Diabetes 51(4), 1102-1109 (2002).

48 Chouchane L, Danguir J, Beji C et al. Genetic variation in the stress protein hsp70-2 gene is highly associated with obesity. Int. J. Obes. Relat. Metab. Disord. 25(4), 462-466 (2001).

49 Zouari Bouassida K, Chouchane L, Jellouli $\mathrm{K}$ et al. Polymorphism of stress protein HSP7O-2 gene in Tunisians: susceptibility implications in Type 2 diabetes and obesity. Diabetes Metab. 30(2), 175-180 (2004).
50 Lee HK, Lee BH, Park SA, Kim CW. The proteomic analysis of an adipocyte differentiated from human mesenchymal stem cells using two-dimensional gel electrophoresis. Proteomics 6(4), 1223 1229 (2006).

51 Zvonic S, Lefevre M, Kilroy G et al. Secretome of primary cultures of human adipose-derived stem cells: modulation of serpins by adipogenesis. Mol. Cell. Proteomics 6(1), 18-28 (2007).

52 Alvarez-Llamas G, Szalowska E, de Vries MP et al. Characterization of the human visceral adipose tissue secretome. Mol. Cell. Proteomics. 6(4), 589-600 (2007).

- One of the few papers focusing on the omental adipose tissue secretome.

53 Klimcakova E, Moro C, Mazzucotelli A et al. Profiling of adipokines secreted from human subcutaneous adipose tissue in response to PPAR agonists. Biochem. Biophys. Res. Commun. 358(3), 897-902 (2007).

54 Maury E, Ehala-Aleksejev K, Guiot Y, Detry R, Vandenhooft A, Brichard SM. Adipokines oversecreted by omental adipose tissue in human obesity. Am. J. Physiol. Endocrinol. Metab. 293(3), E656E665 (2007).

55 Barceló-Batllori S, Gomis R. Proteomics in obesity research. Proteomics Clin. Appl. 3, 263-278 (2009).

Focuses on proteomics in obesity regarding mainly animal models and cultured cells.

\section{Affiliations}

- Belén Peral

Instituto de Investigaciones Biomédicas, Alberto Sols, CSIC \& Universidad

Autónoma de Madrid, Arturo Duperier, 4, E-28029 Madrid, Spain

Tel.: +34915854478

Fax: +34915 854401

bperal@iib.uam.es

- Emilio Camafeita

Unidad de Proteómica, Centro Nacional de Investigaciones Cardiovasculares, CNIC, Madrid, Spain

Tel.: +34914531200

Fax: +34914531265

ecamafeita@cnic.es

José-Manuel Fernández-Real

Department of Diabetes, Endocrinology and Nutrition, \& CIBERobn Fisiopatología de la Obesidad y Nutrición. Hospital Dr. Josep Trueta, Girona, Spain

Tel.: +34972940200

Fax: +34972940270

jmfernandezreal.girona.ics@gencat.cat

- Juan Antonio López

Unidad de Proteómica, Centro Nacional de Investigaciones Cardiovasculares, CNIC, Madrid, Spain

Tel.: +34914531216

Fax: +34 914531265

jalopez@cnic.es 